\title{
SYPHILITIC TESTS IN LATENT AND TREATED SYPHILIS ${ }^{1}$
}

By E. P. Corson-White, M.D., And S. D. W. Ludlum, M.D.

One of the most definite characteristics of syphilis is the complete disappearance of symptoms at certain periods, giving rise to a state of apparently perfect health. This condition described by Ehrlich as a "non-sterilizing immunity" may occur spontaneously or as the result of treatment, and it may last for years. For the safety of the patient the true significance of this period is most important. The continued existence of the organism renders the individual always liable to recrudescence of the infection and possibly to the more serious ravages of para-syphilitic diseases.

During the last few years the Wassermann reaction has been used in the diagnosis of many cases, in the demonstration of the origin of many chronic conditions and in the solution of various other problems. The voluminous literature on the subject shows that in the so-called latent periods of syphilis with apparent health only about thirty per cent. of the cases give negative results. Donath (I) has described what he calls a provocative treatment. This consists in the administration of a short course of mercury or a small dose of salvarsan or neosalvarsan. Apparently this procedure has no effect on the nonluetic serum, but changes about nine per cent. of the negatively reacting serums to positives. Donath himself says the influence of the treatment was similar to that of quinine on latent malaria. It has been explained by others in several ways; namely:

(a) The liberation of endotoxins by the immediate destruction of a small number of spirochetes gives a sudden intense stimulation to antibody formation.

1 Work done in the Laboratory of Neuropathology of the University of Pennsylvania and in the laboratory of the Philadelphia Orthopedic Hospital and Infirmary for Nervous Diseases. 
(b) The dose of spirillicide insufficient to kill the treponema actually stimulates their activity.

(c) The endotoxin liberated from the dead organisms gives rise to that substance in the serum on which the Wassermann reaction depends. Most of the other laboratory methods as the demonstration of the spirochete, or the inoculation of animals, etc., fail at this time.

The luetin test devised by Noguchi, besides the decided advantage of a true specificity, gives the most constant results in tertiary latent and late hereditary cases. The technique of this test consists in the intradermal injection of killed cultures of several strains of Treponema pallidum and a control injection of sterile media. In a syphilitic individual this treatment gives rise to a reddish indurated papule or pustule, often surrounded by an inflammatory area. This inflammation increases for four or five days and then gradually recedes. An inflammatory area may sometimes occur around the control injection. The "Umstimmung" of Lesser. This last is probably due to that increased irritability noticeable in the skin of many syphiiltics, while the first, which is always more severe than the control (when the control shows anything at all), is probably a true allergic condition similar to that found in von Pirquet's reaction for tuberculosis.

The importance of a true interpretation of the position of the apparent clinically well individual with syphilitic history, early led many to use the Wassermann reaction to determine whether the facts described in Colle's and Profeta's laws were due to a real immunity or to a latent infection. Opitz (2) was the first to use it for the study of syphilis in women and newborn children. His results stimulated much careful investigation of the field, by means of the complement fixation tests, demonstration of spirochetic and microscopic examination of tissues.

Plaut (3) in his investigation of the rôle of syphilis in the production of feeble-mindechess studied many complete families including those of paretics and tabetics, finding the majority of the members of these families infected. His series unfortunately included only those in which some mental or nervous defect was present in at least one member of the family. In several families with a positive history present in one parent he found that the children clinically positive gave practically always a positive Wassermann reaction, and the reaction was positive in many 
members of these families that were apparently healthy, but negatively reacting children were also found. These negative results appeared as a rule at one or the other end of the line, although in some cases the negatively reacting child might be between two positives. Knoppelmacher and Lehndorf (4) found that fiftysix per cent. of the mothers of syphilitic children were positive and many more reacted positively if tested within a few months of the babies' birth. Müller and Reich (5) found negative reaction in some children of syphilitic mothers, and Boas and Thomsen (6) in a similar series found that some children which were negative at birth became positive later. Baisch (7) made a very complete study of 140 mothers of children manifestly syphilitic or showing spirochetes in the tissues. He obtained a positive Wassermann reaction in IO2 of these women, and demonstrated the Spirochæta pallida in the maternal position of the placenta in twelve of the negatively reacting mothers. $\mathrm{He}$ concludes for his study, and it certainly appears from most of the evidence, that the mothers of syphilitic children and the children of syphilitic parents are really infected, fifty-two per cent. of the cases giving positive Wassermann results with the original Wassermann technique, a result which can be slightly increased with the one unit or the Noguchi technique. The true position of the negatively reacting children however is not definitely cleared up by the Wassermann reaction.

It is impossible to accurately state the length of a latent period. A positive reaction may be obtained when the resistance of the body is lowered by some cause or other and the latent individual develops an active syphilis.

In treated cases, having once had a positive reaction, the quantitative reaction is the best index of the intensity of disease and of the effect of the treatment.

Citron (8) was the first to study the effect of treatment, finding a loss of the reaction in sixteen per cent. of the cases. The change varies in different individuals, disappearing quickly in some and persisting even after rigorous treatment in others. Irregular and inadequate treatment never gives rise to negative results. There is a tendency in most cases for the reaction to disappear with continued treatment and to reappear if treatment is discontinued. With salvarsan and neosalvarsan most of the reports are studies of the effect of one injection. The most in- 
teresting of these investigations were those of Noguchi and Bronfenbrenner's (9) quantitative study of IO2 serums, before and at weekly intervals after the injection of salvarsan, and Craig's ( Io) analysis of 225 cases according to stage of disease, intensity of reaction and method of treatment. These reports show that the reaction may become negative in from ten to thirty days after the injection, ustally in the third week, and that a large percentage of these negatives relapse. In all cases there is a definite quantitative reduction in the strength of the reaction, more marked in primary and early secondaries, at which stage relapses are less frequent. Still more favorable are the early cases with weak positive reactions. The reduction in the reaction is roughly comparable to the clinical response but is in every case slower.

The tables which we submit are collected from the work of the last two and a half years and include cases from many sources. They are grouped to show primarily the significance of negative reactions in families of infected individuals (Table $I$ ), and in

TABLE I

\begin{tabular}{|c|c|c|c|c|c|}
\hline & No. & Persons & W. R. + & N.R. + & Luetin \\
\hline $\begin{array}{l}\text { Families of syphilitics . . . . . . } \\
\text { TABETICS: }\end{array}$ & I7 & $3 I$ & 26 & 27 & 29 \\
\hline sterile. & 7 & 7 & I & I & 5 \\
\hline c. abortion . . . . . . . . & 4 & 4 & I & I & 2 \\
\hline children abortion.... & I 7 & 47 & $2 I$ & 22 & 22 \\
\hline children....... & 8 & 28 & 9 & I I & II \\
\hline \multicolumn{6}{|l|}{ PARETICS: } \\
\hline sterile. & 4 & 4 & o & o & I \\
\hline abortion $\ldots \ldots \ldots \ldots \ldots$ & 9 & 9 & 4 & 4 & 4 \\
\hline children abortion . . . . . . & 3 & 7 & 4 & 5 & 5 \\
\hline children . . . . . . . . . & 5 & 14 & 2 & 2 & 2 \\
\hline
\end{tabular}

apparently clinically well-treated patients with luetic histories (Table II). Incidentally, to demonstrate the comparative serological value of various methods of treatment (Table III). While many patient were examined, we have included only those in which we were able to make a luetin test and in which we had a record of carefully titrated serum reactions over a period of at least three months. The great majority of these cases were those not yielding quickly to treatment (late secondaries and tertiaries) and the rather high per cent. of positives in the treated cases is due to the fact that the titration was as fine as possible and anything not absolutely negative was counted as positive. From 
these tables it is evident that the negative reactions occur, slightly earlier with the Wassermann technique than with the Noguchi and both give earlier negatives than can be demonstrated with the luetin. Excluding the cases of paresis and tabes in which the

TABLE II

\begin{tabular}{c|c|c|c|c}
\hline \hline Cases & W. R. & N. R. & Time & Luetin \\
\hline 42 & - & - & $3 \mathrm{mo.}$ & $\mathrm{I}-$ \\
27 & - & - & $6 \mathrm{mo.}$ & $\mathrm{I}-3 \pm$ \\
$\mathrm{I} 4$ & - & - & $9 \mathrm{mo.}$ & $5-2 \pm$ \\
$\mathrm{I7}$ & - & - & I yr. & Ix-2 \\
\hline
\end{tabular}

luetin test results were irregular, we found that every form of treatment reduced quantitatively the antibody content of the serum. The reduction is less marked in cases starting treat-

TABLE III

\begin{tabular}{|c|c|c|c|c|c|c|}
\hline Cases & W. R. & Treatment & Stage & W. R. + & N.R. + & Luetin \\
\hline 7 & $\mathrm{x} \times \mathrm{xx}$ & Hg. inc. 3 mo. & 2 dary & 7 av. $x$ & 7 av. $x x$ & 7 \\
\hline II & $\mathrm{xxxx}$ & $\mathrm{Hg}$ inc. $3 \mathrm{mo}$. & 3-ary & II av. $\mathrm{xx}$ & II av. $\mathrm{xx}$ & $I \dot{I}$ \\
\hline I 8 & $\operatorname{xxxx}$ & $\mathrm{Hg}$ : inc. 3 mo. & tabes & $x \operatorname{sav} \cdot x x$ & I sav. $\mathrm{xx}$ & I I \\
\hline 8 & $\operatorname{xxxx}$ & Hg. inc. 3 mo. & paresis & $\operatorname{sav} \cdot \mathrm{xxxx}$ & sav. $\operatorname{xxxx}$ & 6 \\
\hline $\mathrm{I} 2$ & $\mathrm{x}$ & $\mathrm{Hg}$. inc. $3 \mathrm{mo}$. & 2dary & 5 av. $x$ & 5 av. $x$ & I2 \\
\hline 9 & $\mathbf{x}$ & Hg. inc. 3 mo. & 3-ary & 5 av. $x$ & 7 av. $x$ & 9 \\
\hline 5 & $\mathbf{x}$ & $\mathrm{Hg}$. inc. $3 \mathrm{mo}$. & tabes & 3 av. $x$ & 3 av. $x$ & 2 \\
\hline 3 & $\mathrm{xx}$ & $\mathrm{Hg}$. inc. $3 \mathrm{mo}$. & paresis & 3 av. $\mathrm{xx}$ & 3 av. $x x$ & 2 \\
\hline 8 & $\mathrm{xxxx}$ & $606.4 \mathrm{inj}$. & 2dary & 2 av. $x$ & 3 av. $x$ & 6 \\
\hline 8 & $\operatorname{xxxx}$ & $606.4 \mathrm{inj}$ & 3 -ary & 2 av. $x x$ & 5 av. $x x$ & 8 \\
\hline 7 & $\operatorname{xxxx}$ & $606.4 \mathrm{inj}$ & tabes & 5 av. $\mathrm{xxx}$ & $5 \mathrm{av} . \mathrm{xxx}$ & 5 \\
\hline 4 & $\mathrm{xxxx}$ & 606.4 inj. & paresis & 4 av. $x x x$ & 4 av. $\operatorname{xxx}$ & 4 \\
\hline 9 & $\mathrm{xx}$ & 606.4 inj. & 2dary & 0 & I $x$ & 4 \\
\hline 9 & $x$ & $606.4 \mathrm{inj}$ & 3-ary & I av. $x$ & $I x$ & 7 \\
\hline 3 & $\mathrm{x}$ & $606.4 \mathrm{inj}$. & tabes & I av. $x$ & $\ldots \ldots$ & $I$ \\
\hline 9 & $x x$ & $606.4 \mathrm{inj}$. & paresis & 8 av. $x$ & 9 av. $x$ & 3 \\
\hline 3 & $\mathrm{xxxx}$ & $9 \mathrm{r} 4.4 \mathrm{inj}$. & 2 dary & 2 av. $x$ & 2 av. $\mathrm{xx}$ & 3 \\
\hline 7 & $\mathrm{xxxx}$ & 2 & 3-ary & 3 av. $x$ & 3 av. $x$ & 6 \\
\hline 3 & $\mathrm{xxxx}$ & 2 & tabes & 3 av. $x x$ & 3 av. $x x$ & 2 \\
\hline 3 & $\operatorname{xxxx}$ & 2 & paresis & 3 av: $x x x$ & 3 av. $x x x$ & 2 \\
\hline I 2 & $\mathrm{x}$ & 2 & 2 dary & 0 & 2 av. $x$ & 9 \\
\hline 8 & $\mathbf{x}$ & 2 & 3 -ary & 2 av. $x$ & 3 av. $x$ & 5 \\
\hline 2 & $x$ & 2 & tabes & 0 & $\mathbf{r}$ av. $x$ & 2 \\
\hline 2 & $\mathrm{x}$ & 2 & paresis & I av. $x$ & I av. $x$ & 0 \\
\hline I4 & $\mathrm{xxxx}$ & $9 I_{4} \mathrm{Hg}$. & 2dary & 2 av. $x$ & 2 av. $x$ & 8 \\
\hline I7 & $\mathrm{xxxx}$ & $9 \mathrm{I} 4 \mathrm{Hg}$. & 3-ary & 2 av. $x$ & 3 av. $x$ & 8 \\
\hline 8 & $\operatorname{xxxx}$ & $9 \mathrm{I} 4 \mathrm{Hg}$. & tabes & 5 av. $x$ & 7 av. $x$ & 6 \\
\hline 3 & $\mathrm{xxxx}$ & $9 \mathrm{I} 4 \mathrm{Ig}$. & paresis & 2 av. $x$ & $3 \mathrm{av} \cdot \mathrm{xx}$ & I \\
\hline 6 & $\operatorname{xxxx}$ & 9I4-I mo. hg. I mo. & 3-ary & 2 av. $x$ & 2 av. $x$ & 6 \\
\hline 2 & $\mathrm{xxxx}$ & 9r4-I mo. hg. I mo. & tabes & 2 av. $x \times x$ & $2 \mathrm{av} \cdot \mathrm{xxx}$ & 2 \\
\hline 3 & $\mathrm{xxxx}$ & 9I4-x mo. hg. I mo. & paresis & $3 \mathrm{av} \cdot \mathrm{xxx}$ & 3 av. $\mathrm{xxx}$ & 2 \\
\hline 7 & $\mathrm{x}$ & 9r4-I mo. hg. I mo. & 3-ary & I X & 4 av. $x$ & 4 \\
\hline 7 & $\mathbf{x}$ & 9.4-I mo. hg. I mo. & tabes & 3 av. $\mathrm{x}$ & 3 av. $x$ & 7 \\
\hline 3 & $\mathrm{x}$ & lor4-I mo. hg. I mo. & paresis & 2 av. $\mathrm{x}$ & 2 av. $x$ & I \\
\hline
\end{tabular}


ment with strongly positive ( $\mathrm{x} \times \mathrm{xx}$ ) reactions (over two units) of antibody than in those with weak reactions ( $x$ ), that is one half a unit or less. It is much less in late stages than in early stages. Slow reductions are apt to be the rule in paresis and occur not. infrequently in some cases of tabes. Kaplan ( I I ) called attention to this type, calling them the "Wassermann fast tabes." The most marked reduction in the reaction appears in those cases treated with mercury and salvarsan or neosalvarsan, and next in those with salvarsan or neosalvarsan alone. The difference between those two is slight. Salvarsan apparently acts more rapidly than the neosalvarsan, although apparently the neosalvarsan remains longer in the blood than the salvarsan. Mercury gives a slow steady diminution. The serum in patients after long continuation of any type of treatment may show a progressive slowing of the reduction of the antibody content and in some cases even a stationary period.

In conclusion, negative reactions do not necessarily mean absence of syphilitic infection or cure, even although the reaction has been negative for considerable time, as shown in Table II. The luetin test, the specificity of which has up to date never been disproved, is less easily influenced by treatment and is to-day the best criterion of genuine cure or absence of infection in our hands.

\section{BIBLIOGRAPHY}

I. Donath, K. Berl. klin. Wchnschr., I909, No. XLVI, p. 2015.

2. Opitz, E. Med. Klin., I908, No. IV, p. II37.

3. Plaut. "The Wassermann sero-diagnosis of Syphilis in its application to Psychiatry," New York, IgI I.

4. Knoppelmacher, W., and Lehndorf, H. Wien. med. Wchnschr., rgo8, No. LVIII, p. 6Io; Jahrb. f. Kinder., I9Io, No. LXX, p. I56.

5. Müller and Reich. Wien. klin. Wchnschr., I908, No. XXI, p. 282.

6. Boas and Thomsen. Zeitschr. f. Immunitätsforsch., Bd. X, IgI I, p. 337.

7. Baisch, K. Munchen. med. Wchnschr., I909, No. LVI, p. I920.

8. Citron, J. Deutsch. med. Wchnschr., I907, No. XXXIII, p. I 165.

9. Noguchi. "Serum Diagnosis of Syphilis," Philadelphia, IgI2.

Io. Craig, C. F. Arch of Intern. Med., I9I I, No. VIII, p. 395.

II. Kaplan. Jour. Amer. Assn., I@I3. 